\title{
Effect of Anti-Tuberculous Therapy on Uveitis Associated with Latent Tuberculosis
}

Sydney-London Latent Ocular TB Study Group

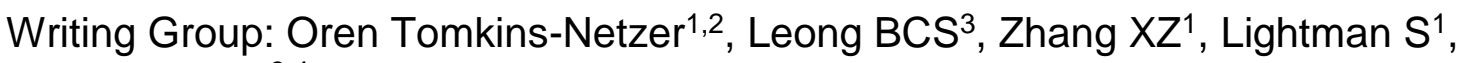
McCluskey $\mathrm{PJ}^{3,4}$.

Members: Oren Tomkins-Netzer ${ }^{1,2}$, Leong $\mathrm{BCS}^{3}$, Lee $\mathrm{AJ}^{3,4,5}$, Zhang $X Z^{1}$, Leahy $\mathrm{K}^{3}$, Zagora $S^{3}$, Younan $\mathrm{C}^{3,4,6}$, Fung $\mathrm{AT}^{3,6,7}$, Nguyen $\mathrm{PV}^{3}$, Lertsumitkul $\mathrm{S}^{3,5}$, Lightman $\mathrm{S}^{1}$, McCluskey $\mathrm{PJ}^{3,4}$.

${ }^{1}$ UCL/Institute of Ophthalmology and Moorfields Eye Hospital London UK

2 Technion, Institute of Technology, Bnai Zion Medical Center, Haifa, Israel

${ }^{3}$ Save Sight Institute, Sydney Medical School, Sydney, Australia

4 Sydney Eye Hospital, Sydney, Australia

${ }^{5}$ Department of Ophthalmology, Liverpool Hospital, Sydney, Australia

6 Department of Ophthalmology, Westmead Hospital, Sydney, Australia

${ }^{7}$ Faculty of Medicine and Health Sciences, Macquarie University Hospital, Australia

* Corresponding author

Dr. Oren Tomkins-Netzer

Institute of Ophthalmology, University College of London,

162-165 City Road

London EC1V 2PD

email: o.tomkins-netzer@ucl.ac.uk

Tel: +442075662266

Fax: +442072519350

Short title: Anti-Tuberculous therapy and uveitis associated with latent TB 


\section{Introduction}

Tuberculosis (TB) with and without associated human immunodeficiency virus (HIV) infection is a worldwide problem and a major concern for the world health organisation (WHO). In 2014 alone 9.6 million people were thought to have been infected with TB globally and 1.1 million HIV negative people died from TB. ${ }^{1}$ With migration and travel, all countries are affected with varying rates of infection with areas of higher rates where migrant groups settle. ${ }^{2}$ Latent TB occurs when individuals have been exposed to TB in the past but did not receive anti-TB treatment (ATT), and have remained systemically well.

Recent migration from TB endemic countries and long duration of exposure are risk factors for latent TB and an associated positive QuantiFERON-TB Gold test (Qiagen, Chadstone, Victoria, Australia, QFT®). ${ }^{3}$ Latent TB carries a risk of about $10 \%$ of reactivation over a person's lifetime although this may vary with location. ${ }^{4}$ With the population size involved, widespread community treatment with ATT for latent TB is difficult, although in selected high risk groups it has been feasible and successful. ${ }^{5} \mathrm{~A}$ further important finding is that latent TB is associated with an increased risk of developing cancer. ${ }^{6}$

If a patient has evidence of active TB infection, such as pulmonary signs and symptoms and an abnormal chest roentgenogram (CXR) with radiologic signs of active TB infection or clinically evident extra-pulmonary involvement, treatment is indicated. Uveitis associated with evidence of active infection may indicate direct ocular involvement by TB organisms. ${ }^{7-9}$ In other patients with severe vision threatening uveitis where systemic corticosteroids and steroid-sparing agents are 
required, ATT may be indicated to prevent reactivation of latent TB, rather than to treat active TB related uveitis. ${ }^{10}$

It is not uncommon when investigating patients with uveitis, that there is no identifiable systemic or ocular disease and that the only positive test is a tuberculin/QFT ${ }^{11} .{ }^{11}$ In such patients, the question arises as to whether the uveitis is related to latent TB or not and whether ATT would be beneficial.

Recent studies in India suggest that in patients with vision threatening uveitis with no identifiable cause who have latent TB, the recurrence rate of the uveitis is greatly reduced with concomitant TB and uveitis treatment - recurrence rate $46.53 \%$ versus $15.74 \%$ in all types of uveitis. ${ }^{12}$ Similar findings have been demonstrated by studies in Singapore ${ }^{13}$, the Netherlands ${ }^{11}$ and the United Kingdom..$^{14,15}$ India and Singapore have intermediate or high population rates of TB infection and all of these studies included some patients with evidence of active TB on CXR in addition to patients with latent TB.

The current study examines the clinical features and outcomes of patients presenting with uveitis who have no evidence of any systemic or ocular disease causing their uveitis and who have latent TB. The study also assesses the effect of ATT on uveitis outcome.

\section{Methods}

This is a retrospective cohort study of patients with uveitis associated with latent TB attending uveitis clinics in London (UK, ethical approval for data collection LIGS10201) and Sydney (Australia, LNR/15/POWH/432) between the years 2007- 
2015. The patient inclusion criteria are summarised in table 1 and included a diagnosis of latent-TB, based on a positive tuberculin or $\mathrm{QFT}^{\circledR}$ test without evidence of active infection or having received ATT. Children from endemic regions for TB are regularly screened for TB and treated as needed. In our cohort there was no evidence of previous testing for TB, suggesting that those who migrated from endemic regions, did so as adults. The presence of a Ghon focus on CXR was not considered evidence of active TB. We considered patients to have uveitis associated with latent TB if, following a complete systemic review and targeted blood investigations (eg toxoplasma serology, angiotensin converting enzyme, antinuclear antibodies, syphilis serology, complete blood count, blood electrolytes, urine analysis, liver enzymes and other as appropriate), there was no other identifiable cause for their uveitis, apart from TB. Patients were excluded if there was another identifiable cause for their uveitis on history, examination or investigation. The anatomical location of ocular involvement was based on clinical assessment at the time of diagnosis. A diagnosis of occlusive vasculitis was based on the presence of corresponding clinical findings such as vascular sheathing, retinal or vitreous hemorrhages and areas of capillary non-perfusion on fluorescein angiography.

Uveitis was categorised anatomically using the SUN criteria. ${ }^{16}$ Ocular treatment was given, appropriate for the anatomical location of the inflammation. Patients were also assessed by respiratory physicians, where further investigations were performed and all patients were offered ATT. ${ }^{12}$ ATT consisted of four drugs: ethambutol, rifampicin, isoniazid and pryazinamide for two months followed by rifampicin and isoniazid only for a further four months, making for a total treatment time of at least six months, based on clinical response. ${ }^{17,18}$ This treatment regimen was prescribed due to either the need for combination immunosuppressive therapy or the potential for escalation 
of immunosuppressive treatment to treat vision threatening uveitis, both of which may result in reactivation of TB. Patients continued treatment with corticosteroids and steroid-sparing immunosuppressive drugs for their uveitis during ATT treatment. Additional ocular management including intraocular pressure lowering medications, vitrectomy for vitreous haemorrhage, argon laser photocoagulation to ischaemic retina causing neovascularisation, bevacizumab for secondary choroidal neovascularisation and cataract surgery, was instituted as needed.

Patient demographics and clinical information including: length of follow-up, anatomical diagnosis of uveitis, treatment, response to treatment, duration of time to and number of recurrences of inflammation, ocular complications and best corrected visual acuity (BCVA) at presentation and final follow-up were collected. Recurrence of inflammation was defined as any intra-ocular inflammation occurring in the same eye, six or more months after uveitis was controlled. BCVA was recorded using Snellen charts and converted to early treatment diabetic retinopathy study (ETDRS) letters for statistical analysis, using conventional conversion tables. ${ }^{19}$ Patients born in the Indian sub-continent, Middle East, South East Asia, China, Africa or the Pacific islands, were considered to be from TB endemic regions. ${ }^{1}$

\section{Statistical methods}

Information from cases with bilateral ocular involvement was adjusted using appropriate statistical methods. ${ }^{20}$ The change in mean BCVA throughout follow-up was compared using generalized estimating equation, adjusting for time of follow-up and correlations between two treated eyes of the same patient. Multivariate analysis and odds ratios $(\mathrm{OR})$ with $95 \%$ confidence intervals $(\mathrm{Cl})$ for recurrence of disease 
was calculated using a Cox regression model while adjusting for correlations between both eyes of the same patient and accounting for the variable follow-up. Only factors with a significance level of at least 0.1 on univariate analysis were included in the multivariate model. In order to adjust for the increased use of ATT in non-AU cases, both these factors were included in the multivariate analysis. The Kaplan-Meier estimator was used to examine survival to the recurrence of ocular inflammation, and groups were compared using a log-rank test. SPSS (version 23, IBM, USA) was used for all analyses. The accepted level of significance for all tests was $\alpha \leq 0.05$. Continuous data are presented as means \pm standard error of the mean (SEM).

\section{Results}

This study included 199 eyes of 129 patients (46.5\% female) diagnosed with uveitis associated with latent TB (Table 2). There were no differences in the demographics or clinical features of the patients seen at each centre other than some small differences in ethnicity reflecting the different geographic locations of the two centres. (data not shown). The patients were therefore analysed as a single study population. Evidence of exposure to tuberculosis was based on a positive tuberculin test (induration $>10 \mathrm{~mm})(\mathrm{n}=60)$ and/or a positive $\mathrm{QFT}^{\circledR}(\mathrm{n}=93$, mean titre value$6.0 \pm 0.7 \mathrm{IU} / \mathrm{mL}$ ). There was no difference in the positive $\mathrm{QFT}^{\circledR}$ levels across the different clinical types of uveitis $(p=0.2)$. Mean age at diagnosis with uveitis was 43.9 \pm 1.3 years (range 5-77), with a median follow-up time of 29 months (range 6287 months). Patients in the cohort were more likely to originate from a TB endemic region $(n=111,86.0 \%)$. Twenty-seven patients $(20.9 \%)$ had evidence of a healed 
Ghon focus on CXR and 102 (79.1\%) had a normal CXR. Non-anterior ocular involvement occurred in $77.9 \%$ of eyes $(n=155)$, most commonly as occlusive vasculitis ( $n=58,29.2 \%)$ or intermediate uveitis $(n=54,27.1 \%)$. At the time of uveitis diagnosis mean BCVA was $82.8 \pm 1.7$ letters. Eighty-nine patients (69\%) received ATT, of whom 87 (97.8\%) suffered no serious side effects and completed a full course of 6 months treatment. There was no difference in the baseline characteristics of patients who received ATT and those who did not . Corticosteroids were used to treat $93.4 \%$ of eyes (69.5\% topical; $15.2 \%$ periocular and $56.9 \%$ systemic corticosteroids), with 180 eyes (90.5\%) achieving a resolution of the intraocular inflammation. Patients receiving ATT were more likely to receive systemic corticosteroids (66.4\% vs. 33.3\%, $\mathrm{p}<0.001)$. Throughout follow-up, second-line immunosuppressive agents were given to treat 10 patients (18 eyes), in four eyes prior to or at the time ATT was started. Patients receiving ATT were not more likely to be treated with second-line immunosuppression (9.3\% vs. $2.6 \%, p=0.27)$. Ocular complications, following uveitis diagnosis, developed in 71 eyes (35.7\%), most commonly cystoid macular edema $(n=19,9.5 \%)$ and cataract $(n=18,9 \%)$.

Over the period of follow-up, the mean change in BCVA following treatment was 4.5 \pm 1.4 letters. There was no difference in the mean change in BCVA between eyes of patients treated with ATT and those who were not $(5.3 \pm 1.5$ vs. $3 \pm 3.1$ letters respectively, $p=0.16)$, nor between those treated with systemic corticosteroids and those who were not (6.73 \pm 1.8 vs. $2 \pm 2.1$ letters, respectively, $p=0.27)$. Among eyes with moderate vision loss (MVL, 20/200<BCVA <20/40, $n=96$ ) or severe vision loss (SVL, BCVA $\leq 20 / 200, n=44$ ) at diagnosis, treatment with systemic corticosteroids resulted in a significant improvement in BCVA in both patients with MVL $(8.9 \pm 3.5$, $\mathrm{p}<0.001)$ and SVL $(22.9 \pm 6.5, \mathrm{p}<0.001)$. No effect on final BCVA occurred with ATT. 
The proportion of eyes that gained 15 letters was the same in those treated with ATT (22\%) and those that did not receive ATT (19\%, OR $0.9795 \% \mathrm{Cl} 0.47-2.0, \mathrm{p}=0.9)$. There no difference in the proportion of eyes that gained 15 letters between those treated with systemic corticosteroids and those that were not $(27.7 \%$ vs. $12.9 \%$, OR $1.3495 \% \mathrm{Cl} 0.66-2.72, \mathrm{p}=0.4)$. There was also no relation between treatment with either ATT or corticosteroids and the chance of avoiding severe vision loss, defined as loss of 15 letters or more.

During follow-up all patients were able to stop uveitis treatment, but 68 eyes (34.9\%) had a recurrence of ocular involvement with a mean of $2.3 \pm 0.36$ recurrences $(0.64 \pm 0.08$ recurrences per-year). The risk factors for disease recurrence were identified using multivariate analysis (Table 3). Eyes of patients receiving ATT were half as likely to develop a recurrence as those not receiving ATT (29.5\% vs. $48.2 \%$, OR $0.5,95 \% \mathrm{Cl} 0.31-0.83, \mathrm{p}=0.006)$. When examined as a single factor, eyes with non-AU disease were less likely to develop disease recurrence. However, once adjusting for other factors, including use of ATT it was not significant on multivariate analysis. There was no increased risk of recurrence among patients from TB endemic regions $(35.7 \%$ vs. $29.6 \%$, OR $1.27,95 \% \mathrm{Cl} 0.61-2.66, \mathrm{p}=0.53)$, between eyes treated with or without systemic corticosteroids ( $35.2 \%$ vs. $35.3 \%$, OR 0.78 , $95 \% \mathrm{Cl}$ 0.48-1.27, $\mathrm{p}=0.32$ ) or between eyes treated with or without second-line immunosuppression agents ( $27.8 \%$ vs. $35.7 \%$, OR $0.44,95 \% \mathrm{Cl} 0.62-3.88, \mathrm{p}=0.34)$. Estimated median time to first recurrence was 79 months (95\% $\mathrm{Cl} 45.7-112.3)$, with eyes treated with ATT recurring at an estimated median of 120 months compared with 51 months in eyes not treated with ATT (95\% Cl 20.2-81.8, $p=0.005$, Figure 1A). Eyes with non-AU had a median time to first recurrence of 79 months $(95 \% \mathrm{Cl} 0$ 160.0), significantly longer than eyes with $\mathrm{AU}$ that had an estimated median time of 
56 months $(95 \% \mathrm{Cl} 24.0-88.0, p=0.03$, Figure 1B). There was no difference between eyes treated with systemic corticosteroids and those that were not.

\section{Discussion}

This study characterises and provides evidence of the outcome of uveitis associated with latent TB, and the role of ATT in preventing disease recurrence. Following diagnosis and treatment visual function remained stable throughout follow-up and patients maintained visual acuity. Treatment with ATT halved the risk of recurrence and delayed the onset of the first recurrence.

The clinical features of the uveitis in this study reflect patterns of uveitis previously recognised as commonly occurring in patients with proven or suspected TB related uveitis. In this study, occlusive retinal vasculitis was the most frequent pattern of uveitis. Occlusive retinal vasculitis has been recognised for more than a century as an important vision threatening ocular association of TB and is frequently called Eales disease. ${ }^{21,22}$ Eales' original disorder is now considered part of a spectrum of occlusive retinal vasculitis associated with variable inflammatory signs in the vitreous and anterior chamber, retinal neovascularisation and vitreous haemorrhage. ${ }^{23,24}$ It can be a severe vision threatening form of uveitis requiring aggressive systemic immunosuppressive therapy and ablative retinal photocoagulation to ischaemic areas of retina when retinal neovascularisation occurs. ${ }^{24}$ 
All anatomical types of uveitis have been reported in patients with latent TB, with intermediate uveitis, AU and multifocal choroiditis common in our study population. Patients were managed using local and/or systemic therapy based on the presence of an inflammatory mediated threat to vision such as macular oedema with decreased vision. Serpiginous choroidopathy was the least common pattern of uveitis. It is recognised that in up to $20 \%$ of patients diagnosed with serpiginous choroidopathy, especially those with atypical clinical features or in patients from TB endemic regions, the uveitis is TB related and requires ATT in addition to immunosuppressive therapy. ${ }^{25,26}$ Patients in this study were more likely to originate from endemic regions.

Where immunosuppressive agents are not required to treat the uveitis, the question arises as to whether to treat the latent TB infection on the grounds that it may be driving the uveitis, ${ }^{27}$ raising the problems related to ATT compliance, drug toxicities and patient unwillingness to take medication for at least six months. There is no consensus on whether ATT in patients with uveitis and latent TB should be given for six, nine months or even longer. In this study, all patients were offered ATT because of the potential escalation of immunosuppressive treatment, which would then require addressing their TB status. AU recurred significantly more frequently and at a shorter latency than the other types of uveitis. This may reflect a higher risk of disease recurrence for $\mathrm{AU}$ or the more chronic nature of non-AU disease.

In some patients there is direct invasion by TB mycobacteria as evidenced by the positive culture or histopathological identification of organisms from involved ocular tissue. In other patients such as those included in the current study, there is no clinical evidence to suggest active TB infection. The pathogenesis of uveitis in these 
patients remains unclear and it is uncertain whether the uveitis is a hypersensitivity response to TB organisms (either local or remote from the eye) or the result of reactivation of latent ocular infection by $M$. tuberculosis. It is theorised that bacterialaden macrophages from alveoli may enter the lymphatics and circulation carrying bacteria to the eye, where the organisms may persist and initiate an immunemediated response. ${ }^{21,28}$ The association between TB and retinal vasculitis, serpiginous choroiditis, and other clinical presentations has been supported by the identification of $M$. tuberculosis DNA by polymerase chain reaction (PCR). ${ }^{28-31}$

The current study provides significant further evidence implicating localised ocular reactivation of mycobacteria as the likely mechanism of TB related uveitis. In this study, there was no clinical evidence supporting a hypersensitivity response in any tissue outside the eye such as skin rash, erythema nodosum or peripheral neuropathy. There was a dramatic reduction in the recurrence rate of uveitis and a significant delay in time to uveitis recurrence, following treatment with ATT. Additionally, there is ample precedent for similar isolated intraocular reactivation of infection without reactivation of systemic infection, in patients with ocular toxoplasmosis and ocular herpetic infection.

The results of this study from a distinct cohort of patients, with evidence of TB exposure and a limited spectrum of uveitis clinical phenotypes, suggest there is a significant role for TB in the ocular disease. The study provides important treatment outcome data that informs clinicians managing patients similar to those in this study. The critical findings are that treatment with ATT was well tolerated, reduced the frequency of uveitis recurrence by $50 \%$ and significantly increased the time to recurrence. These findings have been reported in earlier studies, earlier studies have 
comprised a mixed population of patients with both latent and active TB. ${ }^{12-15}$ The current study is the first to demonstrate the positive effects of ATT in a series of patients with latent TB. In non-TB endemic regions such patients are by far the most common group of patients with TB related uveitis. Treatment for latent TB did not alter the visual outcome and importantly was not associated with any increased risk of severe vision loss. While treatment with ATT is not without systemic side effects, resulting in poor patient compliance in many cases, this positive effect should be considered when considering treatment in patients with uvieits associated with latent TB. The other important finding in this study is that treatment with oral corticosteroids also did not reduce the proportion of patients gaining vision nor did it increase the likelihood of severe vision loss. Among those with a loss of vision, corticosteroids had a positive effect, with significant visual improvement, suggesting a ceiling effect among those with good vision at diagnosis. Steroid therapy did not increase either the risk of recurrence or the time to recurrence of uveitis.

The strength of the study is the large uniform patient cohort with uveitis associated with latent TB. The study is limited by the retrospective collection of data, the variable length of follow-up and missing patient information. There was a high proportion of patients with good initial visual acuity limiting the ability for the study to demonstrate significant improvement in visual acuity. There is recruitment bias as patient inclusion, patient exclusion and treatment were managed by a number of ophthalmologists and TB physicians. It is possible that with longer follow-up more patients might have disease recurrence, which would reduce the difference in rate of recurrence and time to recurrence. With a median follow-up of two and a half years, the results of this study provide useful medium-term outcome data. 
Overall, the results of this study provide strong, positive evidence of the benefit of treating patients with uveitis associated with latent TB with ATT in addition to appropriate local and systemic anti-inflammatory therapy to controlling their uveitis. 


\section{Acknowledgments}

A. No Financial Support was given for this study

B. SL has received consultancy fees from Allergan, GSK, 4Sight, Bayer, AbbVie and Paraxcel and has been on advisory boards of Allergan Bayer AbbVie Santen and GSK.

PMc has received consultancy fees from AbbVie, Santen and Servier.

OTN has received consultancy fees from AbbVie

ZXZ was supported by the University College London Overseas Research Scholarship.

No other authors have any financial interests to disclose.

C. No other acknowledgements are reported. 
1. WHO Global Tuberculosis Report 2015: World Health Organization, 2015.

2. Pareek M, Greenaway C, Noori T, et al. The impact of migration on tuberculosis epidemiology and control in high-income countries: a review. BMC Med 2016;14:48.

3. Dyrhol-Riise AM, Gran G, Wentzel-Larsen T, et al. Diagnosis and follow-up of treatment of latent tuberculosis; the utility of the QuantiFERON-TB Gold In-tube assay in outpatients from a tuberculosis low-endemic country. BMC Infect Dis 2010;10:57.

4. Shea KM, Kammerer JS, Winston CA, et al. Estimated rate of reactivation of latent tuberculosis infection in the United States, overall and by population subgroup. Am J Epidemiol 2014;179(2):216-225.

5. Rennert-May E, Hansen E, Zadeh T, et al. A Step toward Tuberculosis Elimination in a Low-Incidence Country: Successful Diagnosis and Treatment of Latent Tuberculosis Infection in a Refugee Clinic. Can Respir J doi: 10.1155/2016/7980869. 2016.02.24.

6. Su VY, Yen YF, Pan SW, et al. Latent Tuberculosis Infection and the Risk of Subsequent Cancer. Medicine (Baltimore) 2016;95(4):e2352.

7. Agrawal R, Gupta B, González-López JJ,et al. Spectrum of Choroidal Involvement in Presumed Ocular Tuberculosis: Report from a Population with Low Endemic Setting for Tuberculosis. Ocul Immunol Inflamm 2017;25(1):97-104.

8. Gunasekeran DV, Gupta B, Cardoso J, Pavesio CE, Agrawal R. Visual Morbidity and Ocular Complications in Presumed Intraocular Tuberculosis: An Analysis of 354 Cases from a Non-Endemic Population. Ocul Immunol Inflamm doi: 10.1080/09273948.2017.1296580. 2017.03.20.

9. Agrawal R, Gunasekeran DV, Grant R, et al. Clinical Features and Outcomes of Patients With Tubercular Uveitis Treated With Antitubecular Therapy in the Collaborative Ocular Tuberculosis Study (COTS)-1. JAMA Ophthalmol doi: 10.1001/jamaophthalmol.2017.4485. 2017.10.26.

10. Agrawal R, Gupta B, Gonzalez-Lopez JJ, et al. The role of anti-tubercular therapy in patients with presumed ocular tuberculosis. Ocul Immunol Inflamm $2015 ; 23(1): 40-46$.

11. La Distia Nora R, van Velthoven ME, Ten Dam-van Loon NH, et al. Clinical manifestations of patients with intraocular inflammation and positive QuantiFERON-TB gold in-tube test in a country nonendemic for tuberculosis. $\mathrm{Am}$ J Ophthalmol 2014;157(4):754-761.

12. Bansal R, Gupta A, Gupta V, et al. Role of anti-tubercular therapy in uveitis with latent/manifest tuberculosis. Am J Ophthalmol 2008;146(5):772-779.

13. Ang M, Hedayatfar A, Wong W, Chee SP. Duration of anti-tubercular therapy in uveitis associated with latent tuberculosis: a case-control study. $\mathrm{Br} J$ Ophthalmol 2012;96(3):332-336.

14. Sanghvi C, Bell C, Woodhead M, et al. Presumed tuberculous uveitis: diagnosis, management, and outcome. Eye (Lond) 2011;25(4):475-480.

15. Agrawal R, Gonzalez-Lopez JJ, Nobre-Cardoso J, et al. Predictive factors for treatment failure in patients with presumed ocular tuberculosis in an area of low endemic prevalence. Br J Ophthalmol 2016;100(3):348-355.

16. Jabs DA, Nussenblatt RB, Rosenbaum JT. Standardization of uveitis nomenclature for reporting clinical data. Results of the First International Workshop. Am J Ophthalmol 2005;140(3):509-516.

17. Pollett S, Banner P, O'Sullivan MV, Ralph AP. Epidemiology, Diagnosis and Management of Extra-Pulmonary Tuberculosis in a Low-Prevalence Country: $A$ 
Four Year Retrospective Study in an Australian Tertiary Infectious Diseases Unit. PLoS One 2016;11(3):e0149372.

18. Centers for Disease C, Prevention, American Thoracic S. Update: adverse event data and revised American Thoracic Society/CDC recommendations against the use of rifampin and pyrazinamide for treatment of latent tuberculosis infection-United States, 2003. Morb Mortal Wkly Rep 2003;52(31):735-739.

19. DICOM Standards Committee. Ophthalmic Refractive Reports Use Cases (Informative). 2013.

20. Tomkins-Netzer O, Lightman S, Drye L, et al. Outcome of Treatment of Uveitic Macular Edema: The Multicenter Uveitis Steroid Treatment Trial 2Year Results. Ophthalmology 2015;122(11):2351-2359.

21. Gupta V, Gupta A, Rao NA. Intraocular tuberculosis--an update. Surv Ophthalmol 2007;52(6):561-587.

22. Biswas J, Sharma T, Gopal L, et al. Eales disease-an update. Surv Ophthalmol 2002;47(3):197-214.

23. Biswas J, Ravi RK, Naryanasamy A, et al. Eales' disease - current concepts in diagnosis and management. J Ophthalmic Inflamm Infect 2013(1);3:11.

24. Das T, Pathengay A, Hussain N, Biswas J. Eales' disease: diagnosis and management. Eye (Lond) 2010;24(3):472-482.

25. Vasconcelos-Santos DV, Rao PK, Davies JB, et al. Clinical features of tuberculous serpiginouslike choroiditis in contrast to classic serpiginous choroiditis. Arch Ophthalmol 2010;128(7):853-858.

26. Nazari Khanamiri $H$, Rao NA. Serpiginous choroiditis and infectious multifocal serpiginoid choroiditis. Surv Ophthalmol 2013;58(3):203-232.

27. Tufariello JM, Chan J, Flynn JL. Latent tuberculosis: mechanisms of host and bacillus that contribute to persistent infection. Lancet Infect Dis 2003;3(9):578590.

28. Biswas J, Therese L, Madhavan HN. Use of polymerase chain reaction in detection of Mycobacterium tuberculosis complex DNA from vitreous sample of Eales' disease. Br J Ophthalmol 1999;83(8):994.

29. Gupta V, Shoughy SS, Mahajan S, et al. Clinics of ocular tuberculosis. Ocul Immunol Inflamm 2015;23(1):14-24.

30. Singh R, Toor P, Parchand S, et al. Quantitative polymerase chain reaction for Mycobacterium tuberculosis in so-called Eales' disease. Ocul Immunol Inflamm 2012;20(3):153-157.

31. Madhavan HN, Therese KL, Gunisha P, et al. Polymerase chain reaction for detection of Mycobacterium tuberculosis in epiretinal membrane in Eales' disease. Invest Ophthalmol Vis Sci 2000;41(3):822-825. 


\section{Figure legends}

Figure 1- Survival to first recurrence of TB related uveitis. (A) in eyes receiving ATT median time to recurrence was 120 months compared with 26 months in eyes with no treatment $(95 \% \mathrm{Cl} 2.7-49.3, \mathrm{p}=0.002)$. (B) Eyes with non-AU had a median time to first recurrence of 79 months $(95 \% \mathrm{Cl} 13.1-144.91)$ compared with 42 months in eyes with $\mathrm{AU}(95 \% \mathrm{Cl} 13.87-70.13, \mathrm{p}=0.004)$. 